\title{
CENTRAL LIMIT THEOREM AND ALMOST SURE CENTRAL LIMIT THEOREM FOR THE PRODUCT OF SOME PARTIAL SUMS
}

\author{
YU MIAO
}

\begin{abstract}
In this paper, we give the central limit theorem and almost sure central limit theorem for products of some partial sums of independent identically distributed random variables.
\end{abstract}

\section{INTRODUCTION}

Let $\left(X_{n}\right)_{n \geq 1}$ be a sequence of independent identically distributed (i.i.d.) positive random variables (r.v.). Recently there have been several studies to the products of partial sums. It is well known that the products of i.i.d. positive, square integrable random variables are asymptotically log-normal. This fact is an immediate consequence of the classical central limit theorem (CLT). This point, up to the knowledge of the author, was first argued by Arnold and Villaseñr [1], who considered the limiting properties of the sums of records. In their paper Arnold and Villaseñr obtained the following version of the CLT for a sequence of i.i.d. exponential r.v.'s $\left(X_{n}\right)_{n \geq 1}$ with the mean equal to one:

$$
\frac{\sum_{k=1}^{n} \log S_{k}-n \log n+n}{\sqrt{2 n}} \stackrel{\mathcal{L}}{\rightarrow} \Phi, \text { as } n \rightarrow \infty,
$$

where $S_{k}=\sum_{j=1}^{k} X_{j}, 1 \leq k \leq n$, and $\Phi$ is a standard normal r.v.. Rempała and Wesołowski [8] have noted that this limit behavior of a product of partial sums has a universal character and holds for any sequence of square integrable, positive i.i.d. random variables. Namely, they have proved the following.

Theorem RW Let $\left(X_{n}\right)_{n \geq 1}$ be a sequence of i.i.d. positive square integrable random variables with $\mathbb{E} X_{1}=\mu, \operatorname{Var} X_{1}=\sigma^{2}>0$ and the coefficient of variation $\gamma=\sigma / \mu$. Then

$$
\left(\frac{\prod_{k=1}^{n} S_{k}}{n ! \mu^{n}}\right)^{1 /(\gamma \sqrt{n})} \stackrel{\mathcal{L}}{\rightarrow} e^{\sqrt{2} \Phi} .
$$

Recently, Gonchigdanzan and Rempała [4] discussed an almost sure limit theorem for the product of the partial sums of i.i.d. positive random variables as follows.

2000 Mathematics Subject Classification. 60F05, 60F15.

Key words and phrases. Central limit theorem; almost sure central limit theorem; products of sums. 
Theorem GR Let $\left(X_{n}\right)_{n \geq 1}$ be a sequence of i.i.d. positive square integrable random variables with $\mathbb{E} X_{1}=\mu>0, \operatorname{Var} X_{1}=\sigma^{2}$. Denote $\gamma=\sigma / \mu$ the coefficient of variation. Then for any real $x$,

$$
\lim _{N \rightarrow \infty} \frac{1}{\log N} \sum_{n=1}^{N} \frac{1}{n} I\left(\left(\frac{\prod_{k=1}^{n} S_{k}}{n ! \mu^{n}}\right)^{1 /(\gamma \sqrt{n})} \leq x\right)=F(x), \text { a.s. }
$$

where $F(\cdot)$ is the distribution function of the r.v. $e^{\sqrt{2} \Phi}$.

For further discussions of the CLT, the author refers to [6, 7]. Zhang and Huang [10] obtained the invariance principle of the product of sums of random variables. It is perhaps worth to notice that by the strong law of large numbers and the property of the geometric mean it follows directly that

$$
\left(\frac{\prod_{k=1}^{n} S_{k}}{n !}\right)^{1 / n} \stackrel{\text { a.s. }}{\longrightarrow} \mu
$$

if only the existence of the first moment is assumed.

Throughout the present paper let $S_{n, k}=\sum_{i=1}^{n} X_{i}-X_{k}$ for all $n \geq 1,1 \leq k \leq n$ and we are interested in the similar results as (1.1) and (1.2).

\section{Central limit theorem}

Theorem 2.1. Let $\left(X_{n}\right)_{n \geq 1}$ be a sequence of i.i.d. positive square integrable random variables with $\mathbb{E} X_{1}=\mu, \operatorname{Var} X_{1}=\sigma^{2}>0$ and the coefficient of variation $\gamma=\sigma / \mu$. Then

$$
\left(\frac{\prod_{k=1}^{n} S_{n, k}}{(n-1)^{n} \mu^{n}}\right)^{1 /(\gamma \sqrt{n})} \stackrel{\mathcal{L}}{\rightarrow} e^{\Phi}
$$

where $\Phi$ is a standard normal r.v.

Proof. Let $Y_{i}=\left(X_{i}-\mu\right) / \sigma, i=1,2, \cdots$, then

$$
\frac{1}{\gamma \sqrt{n}} \sum_{k=1}^{n}\left(\frac{S_{n, k}}{(n-1) \mu}-1\right)=\frac{1}{\sqrt{n}} \sum_{k=1}^{n}\left(\frac{\sum_{i \neq k, i \leq n}\left(X_{i}-\mu\right)}{(n-1) \sigma}\right)=\frac{1}{\sqrt{n}} \sum_{k=1}^{n} Y_{k} .
$$

Therefore from the classical central limit theorem and $\mathbb{E} Y_{i}=0, \operatorname{Var}\left(Y_{i}\right)=1$ for all $i=1,2, \cdots$, we know that

$$
\frac{1}{\gamma \sqrt{n}} \sum_{k=1}^{n}\left(\frac{S_{n, k}}{(n-1) \mu}-1\right) \stackrel{\mathcal{L}}{\rightarrow} \Phi .
$$

Furthermore let $C_{n, k}=S_{n, k} /((n-1) \mu), k=1,2, \cdots$. By the strong law of large numbers it follows that for any $\delta>0, \exists R$ such that,

$$
\mathbb{P}\left(\sup _{n \geq R, 1 \leq k \leq n}\left|C_{n, k}-1\right| \geq \delta\right)<\delta \text {. }
$$


Taking $\delta<1 / 2$, for any $x \in \mathbb{R}$, we have

$$
\begin{aligned}
\mathbb{P}\left(\frac{1}{\gamma \sqrt{n}} \sum_{k=1}^{n} \log \left(C_{n, k}\right) \leq x\right)= & \mathbb{P}\left(\frac{1}{\gamma \sqrt{n}} \sum_{k=1}^{n} \log \left(C_{n, k}\right) \leq x, \sup _{n \geq R, 1 \leq k \leq n}\left|C_{n, k}-1\right| \geq \delta\right) \\
& +\mathbb{P}\left(\frac{1}{\gamma \sqrt{n}} \sum_{k=1}^{n} \log \left(C_{n, k}\right) \leq x, \sup _{n \geq R, 1 \leq k \leq n}\left|C_{n, k}-1\right|<\delta\right) \\
:= & A_{n}+B_{n}
\end{aligned}
$$

and

$$
A_{n} \leq \delta
$$

Next we will control the term $B_{n}$. By the following logarithm:

$$
\log (1+x)=x+\frac{x^{2}}{(1+\theta x)^{2}}
$$

where $\theta \in(0,1)$ depends on $x \in(-1,1)$, we have

$$
\begin{aligned}
& B_{n}=\mathbb{P}\left(\frac{1}{\gamma \sqrt{n}} \sum_{k=1}^{n} \log \left(C_{n, k}\right) \leq x, \sup _{n \geq R, 1 \leq k \leq n}\left|C_{n, k}-1\right|<\delta\right) \\
= & \mathbb{P}\left\{\frac{1}{\gamma \sqrt{n}} \sum_{k=1}^{n}\left(C_{n, k}-1\right)+\frac{1}{\gamma \sqrt{n}} \sum_{k=1}^{n} \frac{\left(C_{n, k}-1\right)^{2}}{\left(1+\theta_{k}\left(C_{n, k}-1\right)\right)^{2}} \leq x, \sup _{n \geq R, 1 \leq k \leq n}\left|C_{n, k}-1\right|<\delta\right\} \\
= & \mathbb{P}\left\{\frac{1}{\gamma \sqrt{n}} \sum_{k=1}^{n}\left(C_{n, k}-1\right)+\left[\frac{1}{\gamma \sqrt{n}} \sum_{k=1}^{n} \frac{\left(C_{n, k}-1\right)^{2}}{\left(1+\theta_{k}\left(C_{n, k}-1\right)\right)^{2}}\right] I\left(\sup _{n \geq R, 1 \leq k \leq n}\left|C_{n, k}-1\right|<\delta\right) \leq x\right\} \\
& -\mathbb{P}\left\{\frac{1}{\gamma \sqrt{n}} \sum_{k=1}^{n}\left(C_{n, k}-1\right) \leq x, \sup _{n \geq R, 1 \leq k \leq n}\left|C_{n, k}-1\right| \geq \delta\right\} \\
:= & D_{n}+F_{n},
\end{aligned}
$$

where $\theta_{k}, k=1, \cdots, n$ are $(0,1)$-valued random variables and $F_{n} \leq \delta$. To estimate the term $D_{n}$, by the following elementary inequality: for $|x|<1 / 2$ and any $\theta \in(0,1)$ it follows that $x^{2} /(1+\theta x)^{2} \leq 4 x^{2}$, then we have

$$
\left[\frac{1}{\gamma \sqrt{n}} \sum_{k=1}^{n} \frac{\left(C_{n, k}-1\right)^{2}}{\left(1+\theta_{k}\left(C_{n, k}-1\right)\right)^{2}}\right] I\left(\sup _{n \geq R, 1 \leq k \leq n}\left|C_{n, k}-1\right|<\delta\right) \leq \frac{4}{\gamma \sqrt{n}} \sum_{k=1}^{n}\left(C_{n, k}-1\right)^{2} \stackrel{\mathbb{P}}{\rightarrow} 0
$$

as $n \rightarrow \infty$. Relation (2.5) is a consequence of the Markov inequality, since for any $r>0$

$$
\mathbb{P}\left(\frac{4}{\gamma \sqrt{n}} \sum_{k=1}^{n}\left(C_{n, k}-1\right)^{2} \geq r\right) \leq \frac{4}{r \gamma \sqrt{n}} \sum_{k=1}^{n} \operatorname{Var}\left(C_{n, k}-1\right)=\frac{4}{r \gamma \sqrt{n}} \frac{n \gamma^{2}}{n-1} \rightarrow 0
$$


Therefore $D_{n} \stackrel{\mathcal{L}}{\rightarrow} \Phi(x)$. For any $x \in \mathbb{R}$, we have

$$
\mathbb{P}\left(\log \left(\frac{\prod_{k=1}^{n} S_{n, k}}{(n-1)^{n} \mu^{n}}\right)^{1 /(\gamma \sqrt{n})} \leq x\right)=\mathbb{P}\left(\frac{1}{\gamma \sqrt{n}} \sum_{k=1}^{n} \log C_{n, k} \leq x\right)=A_{n}+D_{n}+F_{n}
$$

which implies our result since the above discussions.

\section{Almost sure central limit theorem}

In this section we will consider the almost sure central limit theorem as (1.2). Starting with Brosamler [3] and Schatte [9], in the past decade several authors investigated the a.s. central limit theorem and related "logarithmic" limit theorems for partial sums of independent random variables. The simplest form of the a.s. central limit theorem (Brosamler, [3]; Schatte, [9]; Lacey and Philipp, [5]) states that if $X_{1}, X_{2}, \cdots$ are i.i.d. random variables with mean 0 , variance 1 and partial sums $S_{n}=\sum_{i=1}^{n} X_{i}$ then

$$
\lim _{N \rightarrow \infty} \frac{1}{\log N} \sum_{k=1}^{N} \frac{1}{k} I\left(\frac{S_{k}}{\sqrt{k}} \leq x\right)=\Phi(x) \text { a.s., } \forall x,
$$

where $I$ denotes indicator function. Berkes and Csáki [2] extended this theory and show that not only the central limit theorem, but every weak limit theorem for independent random variables, subject to minor technical conditions, has an analogous almost sure version. However under our model we only need the simplest version of (3.1).

Theorem 3.1. Let $\left(X_{n}\right)_{n \geq 1}$ be a sequence of i.i.d. positive square integrable random variables with $\mathbb{E} X_{1}=\mu>0, \operatorname{Var} X_{1}=\sigma^{2}$. Denote $\gamma=\sigma / \mu$ the coefficient of variation. Then for any real $x$,

$$
\lim _{N \rightarrow \infty} \frac{1}{\log N} \sum_{n=1}^{N} \frac{1}{n} I\left(\left(\frac{\prod_{k=1}^{n} S_{n, k}}{(n-1)^{n} \mu^{n}}\right)^{1 /(\gamma \sqrt{n})} \leq x\right)=F(x), \text { a.s. }
$$

where $F(\cdot)$ is the distribution function of the r.v. $e^{\Phi}$.

Proof. Let $Y_{i}=\left(X_{i}-\mu\right) / \sigma, i=1,2, \cdots$, then $\mathbb{E} Y_{i}=0$ and $\operatorname{Var}\left(Y_{i}\right)=1$ for all $i=1,2, \cdots$ and from (2.2), for any real $x$, we have

$$
\begin{aligned}
& \lim _{N \rightarrow \infty} \frac{1}{\log N} \sum_{n=1}^{N} \frac{1}{n} I\left(\frac{1}{\gamma \sqrt{n}} \sum_{k=1}^{n}\left(\frac{S_{n, k}}{(n-1) \mu}-1\right) \leq x\right) \\
= & \lim _{N \rightarrow \infty} \frac{1}{\log N} \sum_{n=1}^{N} \frac{1}{n} I\left(\frac{1}{\sqrt{n}} \sum_{k=1}^{n} Y_{k} \leq x\right)=\Phi(x) \quad \text { a.s. }
\end{aligned}
$$


Note that in order to prove (3.2) it is sufficient to show that for any real $x$,

$$
\lim _{N \rightarrow \infty} \frac{1}{\log N} \sum_{n=1}^{N} \frac{1}{n} I\left(\frac{1}{\gamma \sqrt{n}} \sum_{k=1}^{n} \log \frac{S_{n, k}}{(n-1) \mu} \leq x\right)=\Phi(x), \quad \text { a.s. }
$$

To this end let, as before, $C_{n, k}=S_{n, k} /((n-1) \mu)$ and note that by the law of the iterated logarithm we have for $n \rightarrow \infty$,

$$
\max _{1 \leq k \leq n}\left|C_{n, k}-1\right|=O\left(\left(\frac{\log \log n}{n}\right)^{1 / 2}\right) \quad \text { a.s. }
$$

Since for $|x|<1$ we have $\log (1+x)=x+R(x)$ with $\lim _{x \rightarrow 0} R(x) / x^{2}=1 / 2$, thus

$$
\begin{aligned}
& \left|\sum_{k=1}^{n} \log C_{n, k}-\sum_{k=1}^{n}\left(C_{n, k}-1\right)\right| \ll \sum_{k=1}^{n}\left(C_{n, k}-1\right)^{2} \\
& \ll \sum_{k=1}^{n} \frac{\log \log n}{n} \ll \log \log n \log n \text { a.s. }
\end{aligned}
$$

where "«" denote the inequality " $\leq$ " up to some universal constant. Hence for almost every $\omega$ and any $\varepsilon>0$ there exists $n_{0}=n_{0}(\omega, \varepsilon, x)$ such that for $n \geq n_{0}$

$$
\begin{aligned}
I\left(\frac{1}{\gamma \sqrt{n}} \sum_{k=1}^{n}\left(\frac{S_{n, k}}{(n-1) \mu}-1\right) \leq x-\varepsilon\right) & \leq I\left(\frac{1}{\gamma \sqrt{n}} \sum_{k=1}^{n} \log \frac{S_{n, k}}{(n-1) \mu} \leq x\right) \\
& \leq I\left(\frac{1}{\gamma \sqrt{n}} \sum_{k=1}^{n}\left(\frac{S_{n, k}}{(n-1) \mu}-1\right) \leq x+\varepsilon\right)
\end{aligned}
$$

thus (3.3) implies (3.4).

\section{ACKNOWLEDGEMENTS}

Here the author is very grateful to the referee for his valuable report and some helpful suggestions.

\section{REFERENCES}

[1] Arnold, B.C., Villaseñr, J.A., The asymptotic distribution of sums of records, Extremes, 1998, 1(3): 351-363.

[2] Berkes, I., Csáki, E., A universal result in almost sure central limit theory. Stoch. Proce. Appl., 2001, 94: 105-134.

[3] Brosamler, G., An almost everywhere central limit theorem. Math. Proc. Cambridge Philos. Soc., 1988, 104: 561-574.

[4] Gonchigdanzan, K., Rempała, G.A., A note on the almost sure limit theorem for the product of partial sums. Appl. Math. Lett., 2006, 19: 191-196. 
[5] Lacey, M., Philipp, W., A note on the almost everywhere central limit theorem. Statist. Probab. Lett., 1990, 9: 201-205.

[6] Lu, X.W., Qi, Y.C., A note on asymptotic distribution of products of sums. Statist. Probab. Lett., 2004, 68(4): 407-413.

[7] Qi, Y.C., Limit distributions for products of sums. Statist. Probab. Lett., 2003, 62(1): 93-100.

[8] Rempała, G.A. and Wesołowski, J., Asymptotics for products of sums and $U$ statistics. Electron. Comm. Probab., 2002, 7: 47-54.

[9] Schatte, P., On strong versions of the central limit theorem. Math. Nachr. 1988, 137: 249-256.

[10] Zhang, L.X., Huang, W., A note on the invariance principle of the product of sums of random variables. arXiv:math.PR/0610515 v1. 17 Oct 2006.

College of Mathematics and Information Science, Henan Normal University, 453007 Henan, China and Department of Mathematics and Statistics, Wuhan University, 430072 Hubei, China.

E-mail address: yumiao728@yahoo.com.cn 